\title{
The influence of number of syllables on word skipping during reading revisited
}

\author{
Denis Drieghe ${ }^{1} \cdot$ Aaron Veldre $^{2} \cdot$ Gemma Fitzsimmons $^{1} \cdot$ Jane Ashby $^{3} \cdot$ Sally Andrews ${ }^{2}$
}

Published online: 15 March 2019

(C) The Psychonomic Society, Inc. 2019

\begin{abstract}
Fitzsimmons and Drieghe (Psychonomic Bulletin \& Review, 18, 736-741, 2011) showed that a monosyllabic word was skipped more often than a disyllabic word during reading. This finding was interpreted as evidence that syllabic information was extracted from the parafovea early enough to influence word skipping. In the present, large-scale replication of this study, in which we additionally measured the reading, vocabulary, and spelling abilities of the participants, the effect of number of syllables on word skipping was not significant. Moreover, a Bayesian analysis indicated strong evidence for the absence of the effect. The individual differences analyses replicate previous observations showing that spelling ability uniquely predicts word skipping (but not fixation times) because better spellers skip more often. The results indicate that high-quality lexical representations allow the system to reach an advanced stage in the word-recognition process of the parafoveal word early enough to influence the decision of whether or not to skip the word, but this decision is not influenced by number of syllables.
\end{abstract}

Readers obtain useful information not only from the currently fixated word but also from the following word in the sentence, which is typically located in the parafoveal area of the visual field. Many insights about parafoveal processing during reading have been obtained using the influential gaze-contingent boundary paradigm (Rayner, 1975), in which the target word in the parafovea is replaced by a preview stimulus until the readers' eyes cross an invisible boundary located at the end of the preceding word. By manipulating the relationship between the preview and the target word, insights can be obtained about the nature of the information that is extracted from parafoveal vision (see Schotter, Angele, \& Rayner, 2012, for a review).

Pollatsek, Lesch, Morris, and Rayner (1992) used the boundary paradigm to determine that readers extracted phonological information from the parafovea. They used four preview conditions: an identical condition (beach was the preview for the target beach); a homophone preview (beech); an orthographic control word that shared an equal number of

Denis Drieghe

d.drieghe@soton.ac.uk

1 School of Psychology, University of Southampton, Southampton SO17 1BJ, UK

2 University of Sydney, Sydney, Australia

3 Central Michigan University, Mount Pleasant, MI, USA letters with the target as the homophonic preview (bench); and an unrelated preview (house). Fixation durations on the target word were shorter following the homophone preview than the orthographic control word. Pollatsek et al. therefore concluded that phonological information was being extracted and integrated across saccades aiding the subsequent recognition of the target word. Similarly, Ashby and Rayner (2004) reported shorter fixation times when syllabic congruent previews (e.g. de $\pi x w$ as a preview for device) were presented compared with syllabic incongruent previews (e.g. $d e v \_\pi x$ as a preview) even though the incongruent preview shared more letters with the target word. Also, syllable priming influenced ERP's as early as $100 \mathrm{~ms}$ (Ashby, 2010), again indicating very early phonological processing.

Fitzsimmons and Drieghe (2011) examined whether the extraction of phonological information from the parafovea occurred early enough to influence the decision of whether or not to skip the target word. Word skipping is a very common phenomenon, with one out of three words not receiving a direct fixation during reading (for a review, see Rayner, 2009). Short words tend to be skipped more often than long words, words are skipped more often from a close than from a far launch site, high-frequency words are skipped slightly more often than low-frequency words, and words that are predictable from the preceding context are also skipped more often than words that are unpredictable. These findings suggest that easier and closer words are skipped more often than more difficult words, indicating that the parafoveal word has at least 
been partially processed when the decision was made to skip or fixate the next word. It could therefore be assumed that if phonological information was extracted from the parafovea as established by Pollatsek et al. (1992), phonologically simple words would be skipped more often than phonologically more complex words. ${ }^{1}$ However, for information extracted from the parafovea to influence the decision of whether or not to skip the next word, it has to be available early enough in the time course of saccade programming - that is, before the saccade target is selected. According to one of the most important models of eye movements during reading, the E-Z Reader model (Reichle, Pollatsek, Fisher, \& Rayner, 1998), the start of saccade programming is estimated to be $125 \mathrm{~ms}$ before the execution of the saccade. In comparison, the time window during which information can be extracted from the parafovea to influence subsequent fixation times on the target word when it does get fixated - as in the Pollatsek et al. studydoes not close until the eyes move to the target word and the updated visual information reaches the reading system. Hence, evidence that phonological information can affect skipping of an upcoming word implicates the very early extraction of phonological information from the parafovea.

Fitzsimmons and Drieghe (2011) manipulated phonological complexity by varying the number of syllables of a critical target word and observed that a monosyllabic word (e.g. grain) was skipped more often than a disyllabic word (e.g. cargo), but that number of syllables did not influence reading times on the target word when it was fixated. They concluded that phonological parafoveal processing did occur and, more specifically, that phonological information was available early enough to influence saccade target selection. Moreover, the differential impact on word skipping and fixation times implied that different mechanisms underlie the decision to skip the next word or finish fixating it. Although the syllable effect on skipping rate in Fitzsimmons and Drieghe's (2011) data was substantial and statistically robust over both participants and items, subsequent studies conducted in the Southampton eye-tracking lab in which the original stimuli were included as filler materials have failed to replicate the finding of increased skipping of a monosyllabic compared with a disyllabic word. In the light of current 'replication crisis' in psychology, a formal replication is required to establish the generality of the finding.

A possible explanation of the contradictory findings is provided by a gaze-contingent boundary study reported by Chace, Rayner, and Well (2005). Similar to Pollatsek et al. (1992), these authors compared parafoveal preview effects for identical previews, homophone previews, orthographic

\footnotetext{
${ }^{1}$ Note that Pollatsek et al. (1992) could not examine this issue. The phonological relatedness of the preview to the target in their experiment was only apparent after the target appeared, which is after the decision of whether or not to skip has been made.
}

controls, and unrelated consonant strings. Their average data failed to replicate Pollatsek et al.'s finding of significantly stronger preview effects for homophone previews compared with orthographic control previews, but analyses categorising participants on the Nelson-Denny Reading Test, which assesses reading comprehension and vocabulary, revealed a significant phonological preview effect in readers with aboveaverage reading skill that was absent in less skilled readers.

To investigate whether Fitzsimmons and Drieghe's (2011) finding of increased skipping of a monosyllabic compared with a disyllabic word depends on reading skill, we conducted a large-scale replication of the original study in which measures of written language proficiency were collected to assess individual differences in 'lexical quality'. Perfetti (2007) introduced this term to refer to the precision and redundancy of skilled readers' orthographic representations of words and the coherent connections to associated phonological and semantic information. High-quality representations afford rapid, automatic lexical retrieval that allows readers to devote their limited attentional resources to comprehension processes (Perfetti, 2007). Investigations of lexical quality have often relied on measures of reading comprehension and vocabulary, like those used by Chace et al. (2005). However, Andrews (2012) argued that, while these measures are a useful index of the efficiency of lexical and semantic retrieval, they need to be complemented by measures of spelling ability to capture the precise, word-specific knowledge that is central to Perfetti's (2007) specification of lexical quality.

Consistent with the view that reading comprehension and spelling ability tap different components of lexical quality, Veldre and Andrews (2015, 2016, see also Veldre, Drieghe, \& Andrews, 2017) have demonstrated differential effects of these two aspects of proficiency on skipping probabilities and fixation durations. Specifically, higher skipping percentages have been shown to be associated almost exclusively with high spelling ability, whereas high reading ability has been shown to be primarily associated with faster reading times. Applied to the influence of number of syllables on the decision to skip the next word, it is possible that only readers with high-quality lexical representations extract phonological information early enough for it to influence saccade target selection during reading.

\section{Method}

\section{Participants}

Ninety-five undergraduate students from the University of Sydney, with normal or corrected-to-normal vision, participated. All began speaking English before the age of 6 years. Participants received partial course credit for compensation. 


\section{Measurements of written language proficiency}

Participants were administered the vocabulary and reading comprehension subsections of the Nelson-Denny Reading Test (Brown, Fishco, \& Hanna, 1993), as well as a 20 -item spelling dictation test and an 88 -item spelling recognition test (Andrews \& Hersch, 2010). Performance and correlations between the tests are reported in Table 1. Published 4-year U.S. college norms for the Nelson-Denny Reading Test showed that total scores had an average corresponding to the 73rd percentile (range 26th-98th percentile), which is in the average-to-high range and puts us in a good position to observe an effect of phonological complexity restricted to high proficiency readers. Scores on the two subsections of the Nelson-Denny test were highly correlated $(r=.72)$, as were the two spelling tests $(r=.76)$. Therefore, these pairs of measurements were averaged to form composite measures of reading ability and spelling ability, respectively. Reading and spelling ability were entered into the analysis separately, as they were only moderately correlated $(r=.45)$.

\section{Materials and design}

The sixty sentences from Fitzsimmons and Drieghe (2011) were presented featuring either the monosyllabic or disyllabic target word (e.g. The workers were quick at loading the grain/ cargo onto the ship) following a Latin square design. The target words were all five-letter words matched on log frequency, number of orthographic neighbours, mean bigram frequency, and predictability (which was low; for details, see Fitzsimmons \& Drieghe, 2011). As in the original experiment, the 60 experimental sentences were combined with 60 filler sentences and were displayed in a pseudorandom order following 12 practice sentences.

\section{Apparatus}

Eye movements were recorded with a desktop mounted SR Research EyeLink 1000 system. Viewing was binocular, but eye movements were recorded from the right eye only.
Sentences were displayed on a single line on a ViewSonic P225fb CRT monitor in size 16 black Courier New font on a light-grey background. The display was approximately $60 \mathrm{~cm}$ from the participants' eye, and at this distance three characters equalled $1.15^{\circ}$ of visual angle. A chin and forehead rest were used to minimize head movements.

\section{Procedure}

Participants were instructed to read silently for comprehension and that occasionally they would be asked a comprehension question about the sentence they had just read. The initial three-point calibration procedure of the eye tracker took approximately 5 minutes and was repeated whenever necessary during the experiment. At the beginning of each trial, a dot appeared at the location of the first letter of the first word. A stable fixation on this dot triggered the presentation of the sentence. Participants pressed a button to indicate they had finished reading the sentence. Comprehension questions were asked after $25 \%$ of the questions (mean accuracy 93\%). Following this task, participants completed the battery of reading and spelling ability assessments. The experiment lasted approximately 1 hour.

\section{Results}

Fixations shorter than $80 \mathrm{~ms}$ that were within one character of a previous or subsequent fixation were combined with that fixation. All other fixations shorter than $80 \mathrm{~ms}$ were removed, as were those fixations whose durations exceeded 1,000 ms. Trials were removed due to calibration error or if the participant blinked immediately before or after fixating the target word. These exclusions left 5,215 trials (91.5\%) in the analysis. Finally, we also removed observations that were three standard deviations away from the mean for each dependent variable and for the launch distance in the skipping analyses.

Five eye-movement measures were calculated. Skipping probability is the probability that the target word did not receive a direct fixation during first pass; first-fixation duration

Table 1 Means, standard deviations, and range of percentage scores for the language proficiency tests and intercorrelations

\begin{tabular}{|c|c|c|c|c|c|c|}
\hline \multirow[b]{3}{*}{ Individual difference measure } & \multirow[b]{3}{*}{ Mean $(S D)$} & \multirow[b]{3}{*}{ Range } & \multicolumn{4}{|l|}{ Correlations } \\
\hline & & & \multicolumn{2}{|c|}{ Nelson-Denny } & \multicolumn{2}{|l|}{ Spelling } \\
\hline & & & Vocabulary & Reading comprehension & Dictation & Recognition \\
\hline Nelson-Denny vocabulary & $80.07(12.49)$ & $50.0-97.5$ & 1.0 & .72 & .36 & .35 \\
\hline Nelson-Denny reading comprehension & $81.94(13.04)$ & $44.7-97.4$ & & 1.0 & .46 & .40 \\
\hline Spelling dictation & $64.58(19.98)$ & $10.0-100$ & & & 1.0 & .76 \\
\hline Spelling recognition & $86.51(8.81)$ & $58.0-98.9$ & & & & 1.0 \\
\hline
\end{tabular}


is the duration of the first fixation on the target word; singlefixation duration is the duration when exactly one fixation was made; gaze duration is the sum of all fixations on the target word before moving to another word; and go-past time is the sum of all the fixations made from the moment the eyes land on the target word until the first fixation to the right of the target word (i.e., including regressions originating from the target word). The means and standard deviations for all the measurements are shown in Table 2.

The data were analysed using (generalised) linear mixed models (LMM) using the lme4 package (Version 1.1-18-1; Bates, Maechler, Bolker, \& Walker, 2015) in R (Version 3.5.2; R Core Team, 2017). Contrasts for the effect of number of syllables were specified as $-.5 / .5$ such that the intercept corresponded to the grand mean and the fixed effects corresponded to the main effects. Unless otherwise noted, log-transformed data yielded the same pattern of statistical significance as the analysis based on the raw data. Therefore, in the interest of transparency of the effect sizes, we report the analysis of the untransformed data. The skipping data were analysed with logistic GLMMs given their binary nature. The random effects structure of the model consisted of slopes for all the fixed effects across subjects and items, including interactions and correlations (see Barr, Levy, Scheepers, \& Tily, 2013), but was trimmed down for those models that either did not converge or had perfect correlations in the random structure (a sign of overparameterisation). Absolute $t$ values equal to or greater than 1.96 were interpreted as significant because for high degrees of freedom, the $t$ statistic in LMMs approximates the $z$ statistic.

For the fixation time analyses, we included as fixed factors number of syllables, reading ability, and spelling ability. For the skipping analyses, we additionally included launch distance measured in characters removed from the space in front of the target word. All analyses included the two-way interactions with number of syllables. Launch distance, reading ability, and spelling ability were entered into the models as meancentred, continuous variables. All the fixed effect estimates are shown in Table 3. The results were straightforward. No significant main effect of number of syllables or interactions which included this factor were observed on any of the eyemovement measures. In skipping percentage, there was a main effect of spelling ability (better spellers skipped more often than poorer spellers), reading ability (better readers skipped
Table 3 Results of the (generalised) linear mixed models. Statistically significant fixed effects are presented in bold

\begin{tabular}{|c|c|c|c|c|}
\hline Measure & Fixed effect & $b$ & $S E$ & $t / z$ \\
\hline \multirow[t]{8}{*}{ Skipping probability } & Intercept & -2.01 & 0.13 & -15.45 \\
\hline & Syllables & -0.04 & 0.09 & -0.41 \\
\hline & Launch distance & -0.42 & 0.02 & -22.31 \\
\hline & Reading ability & 0.29 & 0.13 & 2.17 \\
\hline & Spelling ability & 0.30 & 0.14 & 2.11 \\
\hline & Syll. $\times$ Reading Ab. & -0.13 & 0.09 & -1.42 \\
\hline & Syll. $\times$ Spelling Ab. & 0.10 & 0.10 & 1.00 \\
\hline & Syll. $\times$ Launch Dist. & -0.03 & 0.03 & -0.96 \\
\hline \multirow[t]{6}{*}{ First fixation duration } & Intercept & 232.05 & 2.97 & 78.09 \\
\hline & Syllables & 1.69 & 2.68 & 0.63 \\
\hline & Reading ability & -7.57 & 3.02 & -2.50 \\
\hline & Spelling ability & -0.08 & 3.25 & -0.03 \\
\hline & Syll. $\times$ Reading Ab. & 1.65 & 2.35 & 0.70 \\
\hline & Syll. $\times$ Spelling Ab & 4.19 & 2.55 & 1.64 \\
\hline \multirow[t]{6}{*}{ Single fixation duration } & Intercept & 237.78 & 4.34 & 54.74 \\
\hline & Syllables & 3.31 & 3.31 & 1.00 \\
\hline & Reading ability & -6.31 & 4.50 & -1.40 \\
\hline & Spelling ability & -1.44 & 4.78 & -0.30 \\
\hline & Syll. $\times$ Reading Ab. & 4.97 & 2.61 & 1.91 \\
\hline & Syll. $\times$ Spelling Ab & 3.22 & 2.94 & 1.10 \\
\hline \multirow[t]{6}{*}{ Gaze duration } & Intercept & 248.78 & 3.81 & 65.23 \\
\hline & Syllables & 5.01 & 4.32 & 1.16 \\
\hline & Reading ability & -11.30 & 3.62 & -3.12 \\
\hline & Spelling ability & -0.80 & 3.85 & -0.21 \\
\hline & Syll. $\times$ Reading Ab. & 2.04 & 2.89 & 0.71 \\
\hline & Syll. $\times$ Spelling Ab & 3.64 & 3.23 & 1.13 \\
\hline \multirow[t]{6}{*}{ Go-past times } & Intercept & 257.54 & 4.31 & 59.71 \\
\hline & Syllables & 4.48 & 4.77 & 0.94 \\
\hline & Reading ability & -12.43 & 3.97 & -3.13 \\
\hline & Spelling ability & -3.25 & 4.26 & -0.76 \\
\hline & Syll. $\times$ Reading Ab. & 1.05 & 3.18 & 0.33 \\
\hline & Syll. $\times$ Spelling Ab & 5.08 & 3.64 & 1.40 \\
\hline
\end{tabular}

more than poorer readers), and launch distance (more skipping from close launch sites than from far launch sites). For all the fixation duration measures, there was a main effect of reading ability such that better readers had shorter fixation times than poorer readers did. The only exception was single-fixation duration where there was no main effect of reading ability in

Table 2 Skipping percentage of the target word and the first-fixation duration, single-fixation duration, gaze duration, and go-past time on the target word (standard deviations in parenthesis)

\begin{tabular}{|c|c|c|c|c|c|}
\hline & Skipping \% & $\begin{array}{l}\text { First } \\
\text { fixation (ms) }\end{array}$ & $\begin{array}{l}\text { Single } \\
\text { fixation (ms) }\end{array}$ & $\begin{array}{l}\text { Gaze } \\
\text { duration (ms) }\end{array}$ & $\begin{array}{l}\text { Go-past } \\
\text { time (ms) }\end{array}$ \\
\hline Monosyllabic & $19.5(12.8)$ & $231(29)$ & $236(39)$ & $246(36)$ & $255(39)$ \\
\hline Disyllabic & $20.3(13.9)$ & 234 (29) & $240(44)$ & $252(36)$ & $261(41)$ \\
\hline
\end{tabular}


the analysis of the untransformed data. However, this was the only analysis for which $\log$ transforming the data resulted in a different pattern of statistical significance such that for the logtransformed data there was a main effect of reading ability, indicating shorter fixation times for better readers $(b=-0.03$, $S E=0.02, t=-1.97)$, in line with all the other fixation time measures. None of the other fixed factors or interactions came close to statistical significance in this model.

\section{Bayesian analysis}

Our main finding of the influence of number of syllables on word skipping and fixation times is a null effect. Bayesian analyses were conducted to establish how much evidence the data yielded for the null hypothesis. We carried out the analysis by comparing two models. In the first model, subjects and items are specified as random factors, and the fixed factors consisted of reading and spelling ability, and, additionally, launch distance for the skipping model. The second model additionally included the factor of number of syllables. The analyses were carried out using the BayesFactor package (Morey \& Rouder, 2013) and used the default scale value (0.5) for the Cauchy priors on effect size and 100,000 Monte Carlo iterations. A low Bayes factor $(<1)$ would indicate evidence for the simpler model, and a high Bayes factor $(>1)$ evidence for a model that does include number of syllables. The Bayes factors reported by the analyses were 0.04 for word skipping, 0.04 for first-fixation duration, 0.08 for singlefixation duration, 0.23 for gaze duration, and 0.11 for gopast time. Using the often-cited evidence categories for the Bayes factor, where a Bayes factor smaller than $1 / 3$ constitutes substantial evidence for the null effect, and a Bayes factor smaller than 1/10 strong evidence, our Bayesian analysis indicated strong evidence for the null effect in skipping percentage, first-fixation duration and single-fixation duration, and substantial evidence in gaze duration and go-past time.

\section{Discussion}

Fitzsimmons and Drieghe (2011) observed that readers skipped monosyllabic words more often than disyllabic words and proposed that syllabic information was extracted during parafoveal processing early enough to influence saccade target selection. The current experiment was instigated by failures to replicate this finding in the Southampton lab and constituted a large-scale attempt to examine whether effects of syllabic structure on skipping were limited to readers with high reading and/or spelling abilities. As in the original study, no effect of syllables was observed on the fixation times on the target word. However, contrary to Fitzsimmons and Drieghe, and regardless of individual differences, the effect of number of syllables on word skipping was not significant. Moreover, our
Bayesian analysis provided strong support for the absence of the effect, indicating that the original finding was probably a Type I error. We will discuss the theoretical importance of these results for views on word skipping, on individual differences during reading, and on the relationship between reading skill and phonological parafoveal processing.

The E-Z Reader model (Reichle et al., 1998) posited two phases of word recognition ( $L 1$ and $L 2$ ). Whereas $L 2$ corresponds to full lexical recognition of a word, $L 1$ has been described as a familiarity check - a point at which activity in the lexical system indicates that recognition is imminent. The distinction between $L 1$ and $L 2$ is very important for E-Z Reader, as it allows for a decoupling between the programming of saccades, which starts after $L 1$ has completed, and shifts of attention to the next word, which only occur when $L 2$ has completed and the word is fully identified. However, the distinction between the two phases is theoretically somewhat underspecified. That is, there is no list of variables that would exclusively impact $L 1$ but not $L 2$ or the other way around. Fitzsimmons and Drieghe's (2011) finding that phonological complexity selectively influenced word skipping implied it was a factor that exclusively influenced the $L 1$ phase as conceptualised by the E-Z Reader model, and not the L2 phase. Because of the important implications for refining this influential model it was crucial to establish the reliability of this effect using a larger sample and more rigorous statistical methods. Confirming our concerns, this more powerful study failed to confirm the original finding.

The hypothesis that individual differences in reading or spelling ability might modulate the increased skipping of monosyllabic versus disyllabic words was also not supported. This possibility was inspired by Chace et al.'s (2005) finding that phonological parafoveal processing during reading was restricted to highly skilled readers, as assessed by tests of reading comprehension and vocabulary. However, the eye-movement literature on the relationship between phonological coding and reading skill is somewhat mixed. Chace et al. found stronger phonological preview effects in more skilled readers, but Jared, Levy, and Rayner (1999) argued that poor readers were more likely to activate phonological codes early, whereas better readers relied more on a direct orthography-to-meaning route. Consistent with this argument is the observation of significant phonological parafoveal processing in primary school children, at least in the orthographically transparent language of German (Tiffin-Richards \& Schroeder, 2015). More recently, Jared and O'Donnell (2017) found no influence of reading skill on the reliance on the phonological code of a word. The current results do not inform this debate, as there was no effect of number of syllables for any of our participants. However, it is clear that the extent to which parafoveal phonological processing is modulated by reading proficiency merits further investigation. 
Although the present results provide no evidence that individual differences modulate the effects of phonological complexity, they replicate effects of reading comprehension and spelling ability demonstrated in a number of previous studies of individual differences in eye movements during reading (Slattery \& Yates, 2018; Veldre \& Andrews, 2015, 2016; Veldre et al., 2017): Whereas high reading ability is associated with shorter fixation times and, contrary to previous studies, also with higher skipping rates, high spelling ability is selectively associated with measures of skipping. This finding suggests that the precise lexical representations indexed by high spelling ability specifically enhance the parafoveal processing that contributes to oculomotor decisions.

In conclusion, the current study provides strong evidence that, contrary to Fitzsimmons and Drieghe (2011), there is no influence of number of syllables on word skipping. This indicates that at least as far as phonological complexity operationalised by number of syllables is concerned, this specific parafoveal phonological information is either not extracted early enough to impact on word skipping or is not taken into account. Consistent with previous studies, our results indicate that word skipping is enhanced by precise lexical representations, but found no evidence that lexical proficiency modulates parafoveal effects of phonological complexity.

Open practices statement Our data and R scripts used for the analyses are available on the Open Science Framework at https://osf.io/rceut/.

\section{References}

Andrews, S. (2012). Individual differences in skilled word recognition and reading: The role of lexical quality. In J. Adelman (Ed.), Visual word recognition (Vol. 2). Hove, UK: Psychology Press.

Andrews, S., \& Hersch, J. (2010). Lexical precision in skilled readers: The role of lexical quality. Individual differences in masked neighbour priming. Journal of Experimental Psychology: General, 139, 299-318.

Ashby, J. (2010). Phonology is fundamental in skilled reading: Evidence from ERPs. Psychonomic Bulletin \& Review, 17, 95-100.

Ashby, J., \& Rayner, K. (2004). Representing syllable information during silent reading: Evidence from eye movements. Language \& Cognitive Processes, 19, 391-426.

Barr, D. J., Levy, R., Scheepers, C., \& Tily, H. J. (2013). Random effects structure for confirmatory hypothesis testing: Keep it maximal. Journal of Memory and Language, 68, 255-278.

Bates, D., Maechler, M., Bolker, B., \& Walker, S. (2015). Fitting linear mixed-effects models using lme4. Journal of Statistical Software, $67,1-48$.

Brown, J. I., Fishco, V. V., \& Hanna, G. (1993). Nelson-Denny reading test. Austin, TX: PRO-ED.

Chace, K. H., Rayner, K., \& Well, A. D. (2005). Eye movements and phonological parafoveal preview: Effects of reading skill. Canadian Journal of Experimental Psychology, 59, 209-217.
Fitzsimmons, G., \& Drieghe, D. (2011). The influence of number of syllables on word skipping during reading. Psychonomic Bulletin \& Review, 18, 736-741.

Jared, D., Levy, B. A., \& Rayner, K. (1999). The role of phonology in the activation of word meanings during reading: Evidence from proofreading and eye movements. Journal of Experimental Psychology: General, 128, 219-264.

Jared, D., \& O'Donnell, K. (2017). Skilled adult readers activate the meanings of high-frequency words using phonology: Evidence from eye tracking. Memory \& Cognition, 45, 334-346.

Morey, R. D., \& Rouder, J. N. (2013). Bayesfactor: Computation of Bayes factors for common designs (R Package Version 0.9.12-4.2) [Computer software]. Retrieved from https://cran.r-project.org/web/ packages/BayesFactor/index.html

Perfetti, C. A. (2007). Reading ability: Lexical quality to comprehension. Scientific Studies of Reading, 11, 357-383.

Pollatsek, A., Lesch, M., Morris, R. K., \& Rayner, K. (1992). Phonological codes are used in integrating information across saccades in word identification and reading. Journal of Experimental Psychology: Human Perception and Performance, 18, 148-162.

R Core Team. (2017). R: A language and environment for statistical computing [Computer software]. Vienna, Austria: R foundation for Statistical Computing. Retrieved from https://www.R-project.org/

Rayner, K. (1975). The perceptual span and peripheral cues in reading. Cognitive Psychology, 7, 65-81.

Rayner, K. (2009). Eye movements and attention in reading, scene perception, and visual search. Quarterly Journal of Experimental Psychology, 62, 1457-1506.

Reichle, E. D., Pollatsek, A., Fisher, D. L., \& Rayner, K. (1998). Toward a model of eye movement control in reading. Psychological Review, $105,125-157$.

Schotter, E. R., Angele, B., \& Rayner, K. (2012). Parafoveal processing in reading. Attention, Perception, \& Psychophysics, 74, 5-35.

Slattery, T. J., \& Yates, M. (2018). Word Skipping: Effects of word length, predictability, spelling and reading skill. Quarterly Journal of Experimental Psychology, 71, 250-259.

Tiffin-Richards, S. P., \& Schroeder, S. (2015). Children's and adults' parafoveal processes in German: Phonological and orthographic effects. Journal of Cognitive Psychology, 27, 53-548.

Veldre, A., \& Andrews, A. (2015). Parafoveal preview benefit is modulated by the precision of skilled readers' lexical representations. Journal of Experimental Psychology: Human Perception and Performance, 41, 219-232.

Veldre, A., \& Andrews, A. (2016). Semantic preview benefit in English: Individual differences in the extraction and use of parafoveal semantic information. Journal of Experimental Psychology: Learning, Memory, and Cognition, 42, 837-854.

Veldre, A., Drieghe, D., \& Andrews, A. (2017). Spelling ability selectively predicts the magnitude of disruption in unspaced text reading. Journal of Experimental Psychology: Human Perception and Performance, 43, 1612-1628.

Publisher's note Springer Nature remains neutral with regard to jurisdictional claims in published maps and institutional affiliations. 